\title{
ISS Space Plasma Laboratory: An ISS instrument package for investigating the opening/closing of solar and heliospheric magnetic fields
}

\author{
Edgar A. Bering, III $^{1}$, Gregg Edeen ${ }^{2}$, \\ University of Houston, Houston, Texas, 77204-5005 \\ Spiro K. Antiochos ${ }^{3}$, C. Richard DeVore ${ }^{4}$, Barbara J. Thompson ${ }^{5}$, \\ NASA/ Goddard Space Flight Center, Greenbelt, Maryland, 20771 \\ Mark Carter $^{6}$, Matthew Giambusso ${ }^{7}$, Chris Olsen ${ }^{8}$, Jared Squire ${ }^{9}$, \\ Ad Astra Rocket Company, Webster, Texas, 77598 \\ Benjamin Longmier ${ }^{10}$, \\ University of Michigan, Ann Arbor, Michigan, 48109 \\ Davin Larson $^{11}$, James P. McFadden ${ }^{12}$, \\ University of California, Berkeley, California, 94720 \\ and \\ David M. Smith ${ }^{13}$ \\ University of California, Santa Cruz, California, 95064
}

\begin{abstract}
We describe a proposed laboratory-experiment research program that will answer the fundamental question: What is the role of reconnection in opening and closing the solar magnetic field? While attacking this question, we will also address the most important, longstanding questions on magnetic reconnection, in all contexts: What determines the rate of reconnection, and whether or not it is bursty? How is the released energy partitioned between thermal, kinetic, and particle? Of course, it seems completely contradictory to use a
\end{abstract}

${ }^{1}$ Professor, Physics and ECE, 617 Science \& Research I, Associate Fellow.

${ }^{2}$ Graduate Student, Physics, , 617 Science \& Research I.

${ }^{3}$ Senior Scientist, Heliophysics Sciences Division, Code 674.

${ }^{4}$ Research Astrophysicist, Heliophysics Sciences Division, Code 671.

${ }^{5}$ Research Astrophysicist, Heliophysics Sciences Division, Code 671.

${ }^{6}$ Senior Research Scientist, 141 W. Bay Area Blvd.

${ }^{7}$ Research Scientist, 141 W. Bay Area Blvd.

${ }^{8}$ Senior Research Scientist, 141 W. Bay Area Blvd., Member.

${ }^{9}$ Director of Research, Ad Astra Rocket Company, 141 W. Bay Area Blvd., Member.

${ }^{10}$ Assistant Professor, Aerospace Engineering, 3017 FXB Building, Member.

${ }^{11}$ Staff Scientist, Space Sciences Laboratory, 6 Gauss Way.

${ }^{12}$ Senior Fellow, Space Sciences Laboratory, 6 Gauss Way.

${ }^{13}$ Associate Professor, Physics, Rm 321, Natural Sciences II.

Copyright (C) 2013 by the University of Houston. Published by the American Institute of Aeronautics and Astronautics with permission. 
laboratory experiment to study an open system, because so far all laboratory plasmas have very solid walls. The pioneering feature of our program is that the experiments will be performed on the International Space Station (ISS). Only by going into space can we obtain the open domain that is absolutely essential for studying the observed solar/heliospheric phenomena. We describe a research program that will provide the instrumentation infrastructure, modeling and solar data expertise and initial scientific understanding required to develop the privately funded Aurora electric propulsion package with its VASIMR $^{\circledR}$ VF-200 high powered plasma source into a wall-less, orbiting ISS Space Plasma Laboratory (ISPL) national facility. The VAriable Specific Impulse Magnetoplasma Rocket (VASIMR $^{\circledR}$ ) is a high power electric spacecraft propulsion system, capable of $I_{\mathrm{sp}}$ /thrust modulation at constant power [E. A. Bering, III, et al., "Observations of single-pass ion cyclotron heating in a transsonic flowing plasma," Phys. Plasmas, 17, 043509, doi: 10.1063/1.3389205, (2010).]. The VASIMR ${ }^{\circledR}$ uses a helicon source to generate plasma. The plasma is leaked though a strong magnetic mirror to a second stage. In the second stage, the plasma is energized by a process that uses left hand polarized slow mode waves launched from the high field side of the ion cyclotron resonance. The single pass ion cyclotron heating (ICH) produces a substantial increase in ion velocity. Ad Astra Rocket Company (AARC) is planning to fly a plasma rocket experiment as a major element of the company's "Aurora" electric power and propulsion test platform on the ISS in 2014. The Aurora platform will support a dual-jet magnetic quadrupole $200 \mathrm{~kW}$ version of the VASIMR ${ }^{\circledR}$ plasma rocket (the VF-200). It will consist of two $100 \mathrm{~kW}$ parallel plasma engines with opposite magnetic dipoles, resulting in a near zero-torque magnetic system. The system will be available for basic plasma physics research in parallel with the testing of the VF-200 engine performance as a high power electric propulsion system. The Aurora package would thus become a National Plasma Physics Laboratory (the ISPL) suitable for plasma physics studies in an open, wall free near-Earth orbital laboratory environment. An ISS arm deployed instrument package similar to the Plasma Diagnostics Package used on STS-3 in conjunction with the OSS-1 experiment and STS-51F in conjunction with Spacelab 2 has been proposed to NASA. The Aurora Plasma Diagnostics Package (APDP) will carry Langmuir probes, an RPA, dc magnetometer, plasma wave detectors, Faraday cups, electrostatic analyzers, solid state energetic particle telescope and Ar II and broadband imagers. The studies that will be performed on this revolutionary facility will not only provide ground-truth experimental answers to the questions above, but undoubtedly discover new and unexpected plasma behavior in the unique environment of the ISPL, leading to new understanding of the Sun and Heliosphere.

\section{Introduction}

A defining property of the Sun's atmosphere is that it has no lid: both the plasma and the magnetic field are free to expand outward indefinitely in response to their internal forces. This all-important property is the physical origin for the constant opening and closing of field lines that has been observed by all NASA missions imaging the corona, from Skylab to the Solar Dynamics Observatory (SDO). The dynamical interchange between open and closed has been observed to take place at all time-scales, from quasi-steady streamer expansion and shrinkage ${ }^{1}$, to slow $(\sim 100 \mathrm{~km} / \mathrm{s})$ streamer blowouts and inflows ${ }^{2}$, to explosive CMEs and eruptive flares $(>1000 \mathrm{~km} / \mathrm{s})^{3}$. In fact, the dynamical opening and closing of field may well be the source of the slow solar wind ${ }^{4,5}$. It is widely believed that magnetic reconnection is the basic process underlying all this activity - certainly for the closing of field lines as in streamer inflows and flares ${ }^{6}$. However, the physical properties of the reconnection are far from understood ${ }^{7,8}$ and, consequently, are the subject of study by essentially every present and upcoming NASA Heliophysics mission. For example, the Magnetospheric MultiScale (MMS) mission is dedicated to the in situ study of reconnection.

This paper describes a proposed space simulation laboratory-experiment research program that will answer the fundamental question:

- What is the role of reconnection in opening and closing the solar magnetic field?

While attacking this question, we will also address the most important, long-standing questions on magnetic reconnection, in all contexts: 
- What determines the rate of reconnection, and whether or not it is bursty?

- How is the released energy partitioned between thermal, kinetic, and particle?

Of course, it seems completely contradictory to plan a laboratory experiment to study an open system, because so far all laboratory plasmas have very solid walls. The pioneering feature of our program is that the experiments will be performed on the ISS. Only by going into space can we obtain the open domain that is absolutely essential for studying the observed solar/heliospheric phenomena. This paper describes a planned research program that will provide the instrumentation infrastructure, modeling and solar data expertise and initial scientific understanding required to develop the privately funded Aurora electric propulsion package with its VASIMR ${ }^{\circledR}$ VF-200 high powered plasma source into a wall-less, orbiting International Space Station (ISS) Space Plasma Laboratory (ISPL) national facility. The studies that will be performed on this revolutionary facility will not only provide ground-truth experimental answers to the questions above, but undoubtedly discover new and unexpected plasma behavior in the unique environment of the ISPL, leading to new understanding of the Sun and Heliosphere. The proposed instrumentation infrastructure can and will be used for investigating the plasma parameters and plume physics of future Hall Effect Thrusters (HET) or other advanced propulsion systems, thus building up the Heliophysics science capabilities of the ISS as a national lab in general.

Furthermore, the Heliophysics science community will obtain this unique new facility at only a very small fraction of its true cost. As discussed below, our planned program greatly leverages the enormous investment already made in the VASIMR ${ }^{\circledR}$ and Aurora programs. Moreover, our Team has broad and deep expertise in laboratory experimentation and solar/heliospheric theory/modeling/data exploration. We will design the facility so that it directly addresses the observed solar phenomena.

\section{Background: VASIMR ${ }^{\circledR}$ Project}

\section{A. What is a VASIMR ${ }^{\circledR}$ device?}

The work in this project will take advantage of the Ad Astra Rocket Company's (AARC's) privately funded VASIMR $^{\circledR}$ engines, the VX-200, and the flight VF-200. The VX-200 engine is an operating two stage high power plasma source, as seen in Figure 1,10,11,12,13,14,15,16. The stages can operate independently of each other. The first stage is a $30 \mathrm{~kW}$ helicon plasma source that operates at $\sim 6.78 \mathrm{MHz}$. A convergent-divergent magnetic field leads into the second stage where the ions are accelerated with an ion cyclotron heating (ICH) coupler ${ }^{17}$ capable of injecting $170 \mathrm{~kW}$ of RF power. A peak magnetic field of $\sim 20 \mathrm{kG}$ is located between the first and second stage. The magnetic field profile within the length of the first and second stages is relatively flat. However, the field profile downstream of the second (ICH) stage is a diverging solenoidal end field ${ }^{18}$. This source can produce an argon jet with a total flux of $10^{22}$ ions/s, parallel temperatures in the frame of the beam ranging from 0.5 to $20 \mathrm{eV}$ and bulk flow velocities from 10 to $>50 \mathrm{~km} / \mathrm{s}^{19}$. Pulse lengths of $>30 \mathrm{~s}$ are possible. The VX-200 vacuum chamber is roughly $10 \mathrm{~m}$ long by $4 \mathrm{~m}$ in diameter. This large exhaust region is many times all other scale sizes in the system, such as the final solenoid radius, and the argon gyroradius. While firing, the background pressure remains below $10^{-}$ ${ }^{4}$ torr. The VX-200 field falls to $<5 \mathrm{G}$ within the chamber. Thus the chamber is large enough to simulate the expansion of a plasma jet into a low field, very low pressure background gas in a way that no prior experiment has done. This work will allow us to develop the instrumentation and validated modeling expertise needed to plan for the truly wall-less ISPL experiment. The key point is that the work in this project leverages the very large VASIMR $^{\circledR}$ investment funded by other sources. 


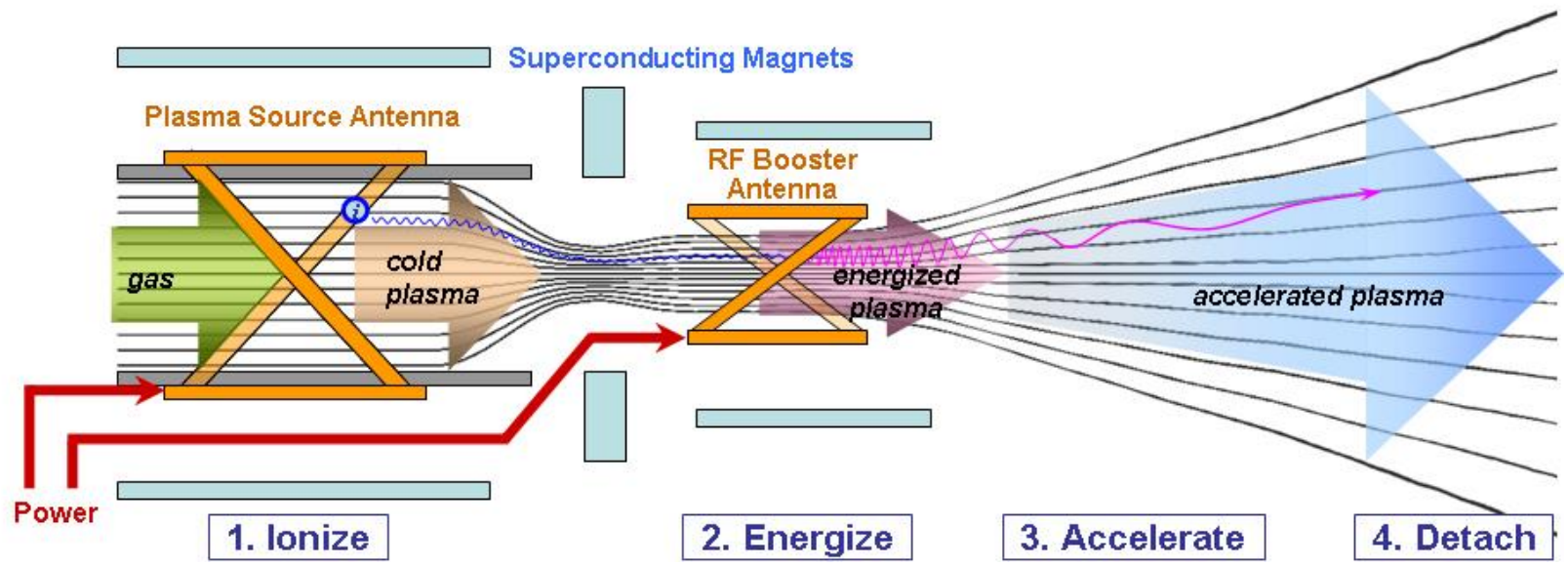

Fig. 1. VX-200 schematic showing magnetic field lines and coupler location

\section{B. Aurora Package and VF-200}

The Aurora is a commercial test-bed that will operate as part of the ISS. The major objective of Aurora is to flight qualify test the performance of Ad Astra's $200 \mathrm{~kW}$ VF-200 VASIMR ${ }^{\circledR}$ engine in the space environment. The Aurora will also enable the testing of other electric propulsion and power technologies in a human-tended space platform. The Aurora has been designated by NASA as a "Pathfinder" external element on the ISS National Laboratory and is being developed by Ad Astra pursuant to its December 8, 2008 Space Act Agreement with NASA to enable the commercial testing of advanced electric propulsion and power systems in support of developing space commerce and exploration requirements. The Aurora will be delivered to the ISS by a commercial launcher and robotic transfer vehicle (TV), such as Orbital Sciences' Cygnus Vehicle or other commercial delivery spacecraft. A rendering of an Aurora concept is shown in Figure 2. Figure 3 shows the hardware installed on the starboard side of the Z1 truss on the ISS.

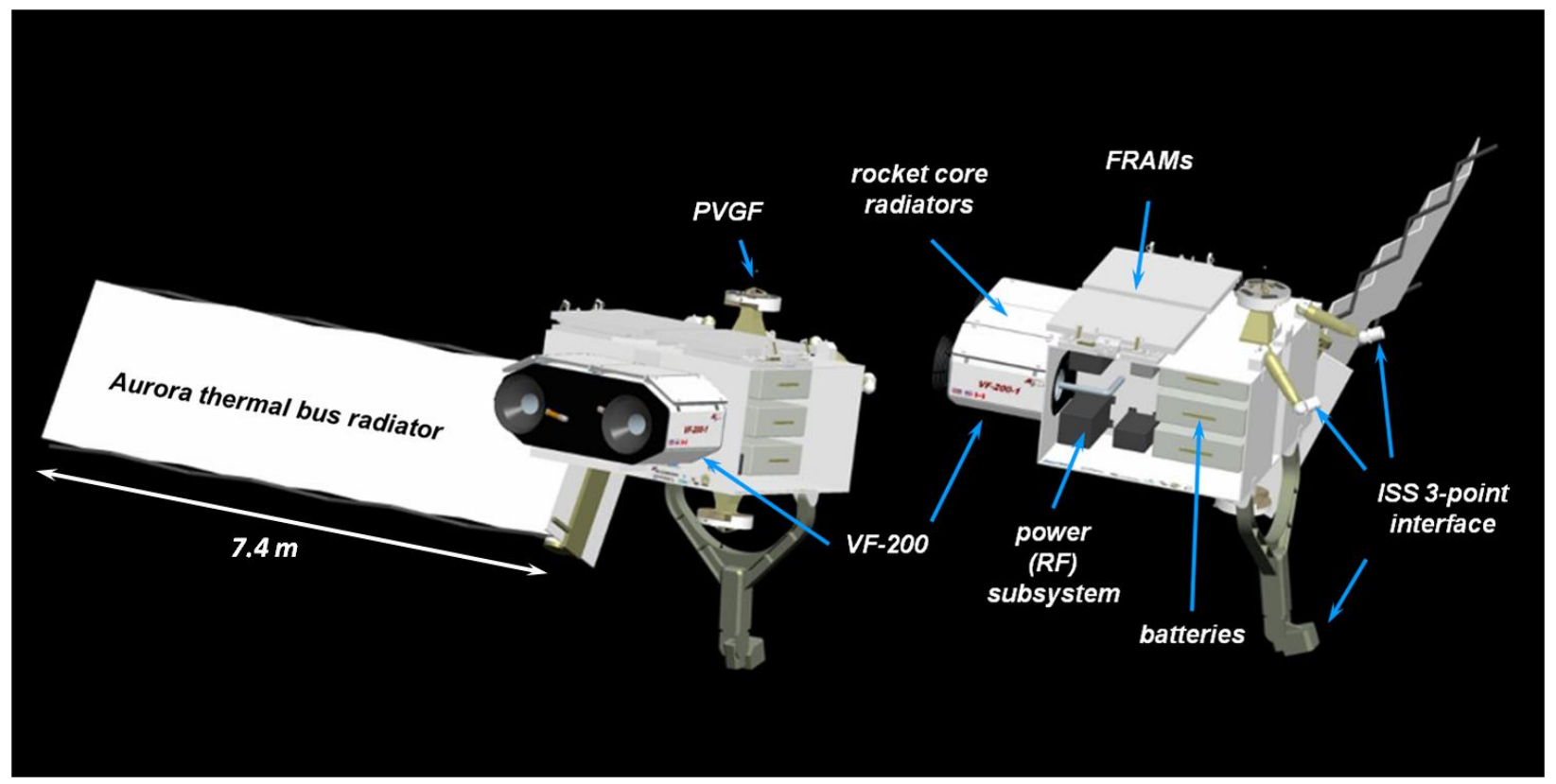

Figure 2: A rendering of the fully deployed Aurora concept. 


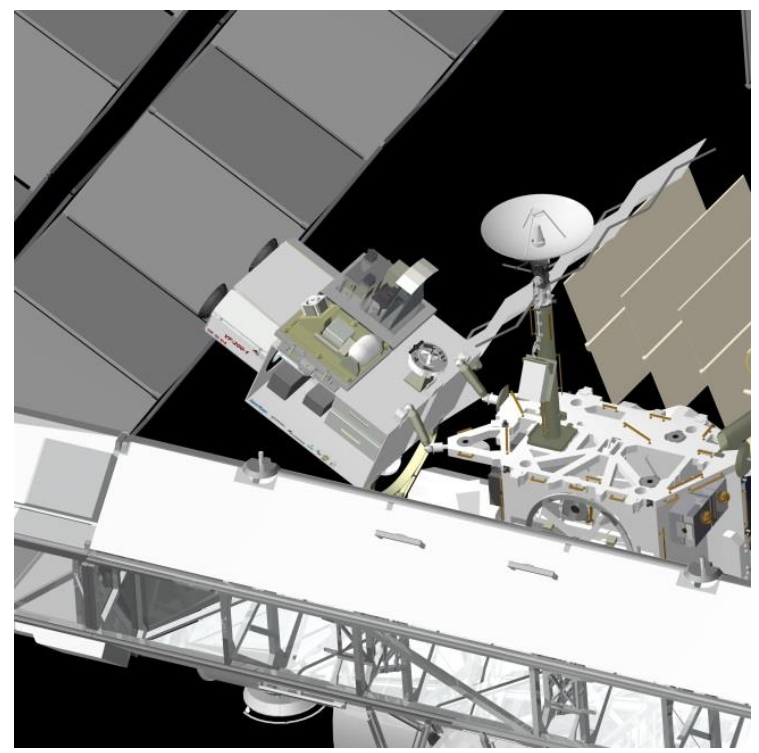

Figure 3: A rendering of the fully deployed Aurora concept in its envisioned position on the International Space Station.

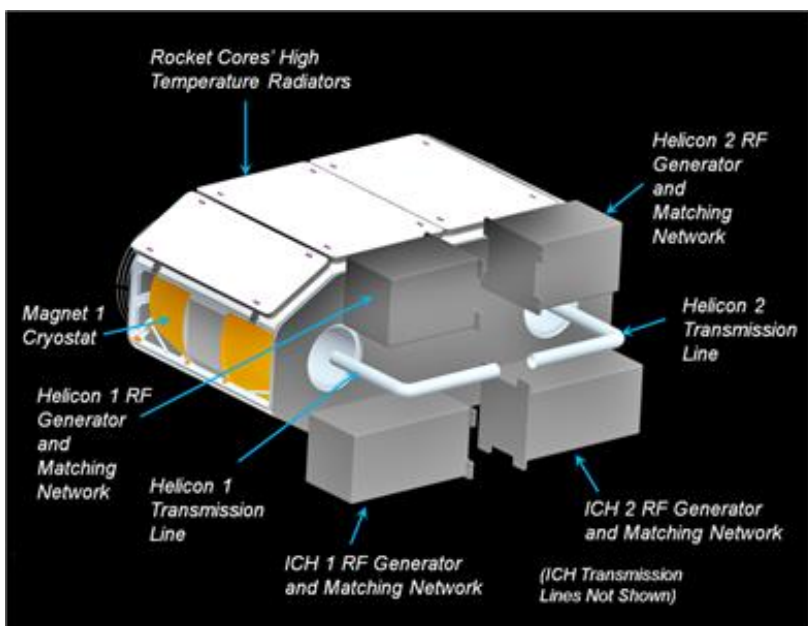

Figure 4: A rendering of a concept of the Aurora Propulsion Element (PRE) shows the general architecture of the VASIMR ${ }^{\circledR}$ VF-200 engine.

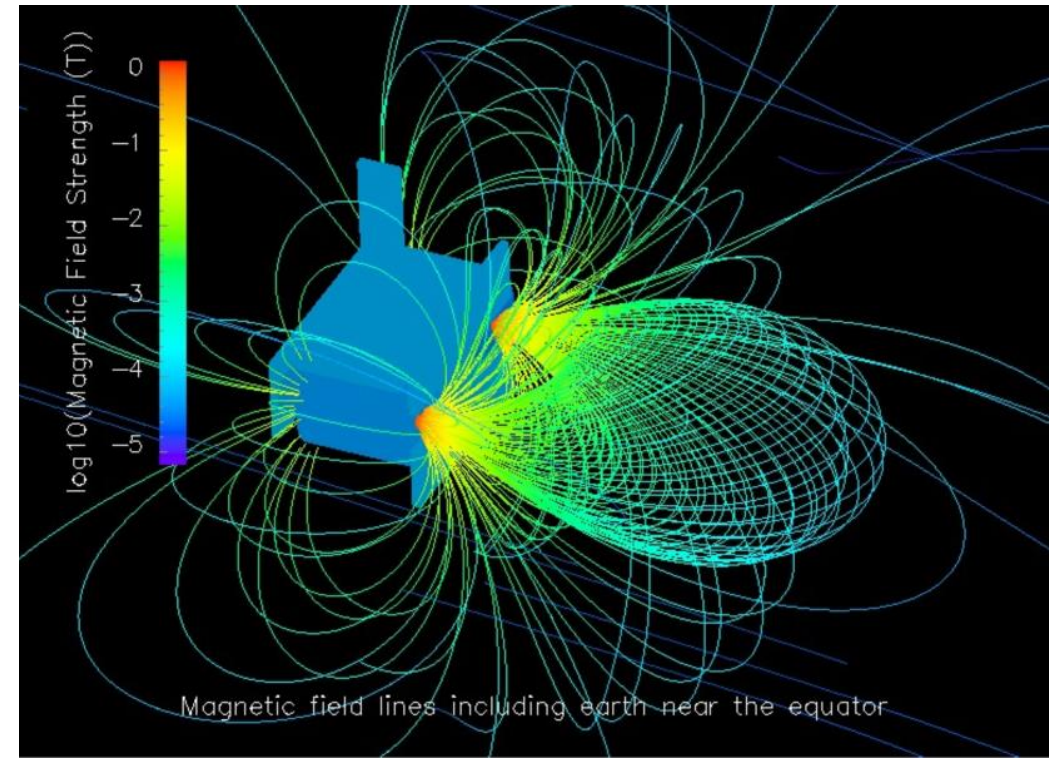

Figure 5. The magnetic field lines of the cancelling dipoles of the two rocket cores of the VF-200-1 in its vacuum configuration. Once the motors are firing, the plasma exhaust will create a helmet-streamerlike configuration.

\section{Aurora Elements}

The Aurora comprises two main elements:

a. Propulsion Element (PRE):

The PRE consists of the VF-200 VASIMR $^{\circledR}$ engine (see Figure 4). Figure 4 illustrates that the VF-200 will be a dual core system consisting of two VASIMR $^{\circledR}$ engines with opposed magnetic polarity that will operate as a magnetic quadrupole with a zero dipole moment. The plasma conditions contained within the physical VF-200 core regions are considered to be proprietary AARC data. The Space Act Agreement provides for the use of the VF-200 exhaust plume as a space borne plasma laboratory environment. The AARC will never consider any aspect of the exhaust plume outside of the VF-200 body to be proprietary. AARC management

strongly supports the use of this exhaust plume as a resource for basic plasma research. The vacuum and plasma magnetic field lines of this device are shown in Figure 5 and 6. In normal operation, both cores will be operated at once, and the plasma pressure will stretch the field lines into an extended configuration that allows the plasma exhaust to produce thrust. The operating state of the engine therefore will consist of a magnetically bipolar streamer containing an internal Y-type current sheet, which separates inner field lines closing between the two nozzles from outer field lines being stretched toward infinity by the plasma flow. 3D reconnection across this sheet will release Ushaped loops of magnetic field and their entrained plasma into the exhaust stream. These are exact analogues to the processes that give rise to helmet streamers, heliospheric current sheets, and ejected plasmoids in the solar corona 
and wind. The project described below is aimed at developing the instruments needed to investigate and understand the detailed physics of the magnetic reconnection taking place in this exhaust of the Aurora rocket on the ISS.

b. Platform Element (PLE): Consists of the components that are required to support the testing of the VF-200 and other propulsion experiments (see Figure 6). A minimum suite of instruments intended to verify nominal VF200 function, monitor Ar ion backflow, and remote-sense the exhaust streamer will be included. The instrumentation required for detailed in situ study of the exhaust streamer is not part of the Aurora PLE design.

As part of the PLE, Aurora will feature a large $(\sim 50 \mathrm{~kW}-\mathrm{hr})$ capacity energy storage module that will enable pulses of up to $200 \mathrm{~kW}$ for periods of up to 15 minutes. Aurora will include several external accommodations, the most important of which is a structural, power, and data interface available to support secondary, commercial highpower $(200 \mathrm{~kW})$ experiments. There will also be external interfaces for auxiliary systems.

Among those potential payloads is a manipulatable Aurora Plasma Diagnostics Package (APDP), whose design is the subject of this project (see $\S$ III), for making the plasma and magnetic-field measurements needed to understand reconnection occurring in the Aurora exhaust streamer.

\section{AARC Lab Experiments}

Test activities will use the new AARC vacuum chamber that is $4.2 \mathrm{~m}$ in diameter with a total internal volume of $150 \mathrm{~m}^{3}$, Figures $7 \mathrm{a}$ and $7 \mathrm{~b}$, and has four 58,000 1/s cryopanels for a total pumping capability of 232,000 1/s on Ar. The vacuum chamber is partitioned into two sections, a rocket section and an exhaust section. The rocket section stays at a space-like vacuum pressure which is lower than the exhaust section while the VX-200 is firing. Also shown in Figure $7 \mathrm{~b}$ is a $2.5 \mathrm{~m}$ by 5 $\mathrm{m}$ translation stage that now carries a suite of plasma diagnostics for exhaust characterization. The translation stage uses 2 independent ball screws and is driven by vacuum compatible stepper motors which yield a positional resolution

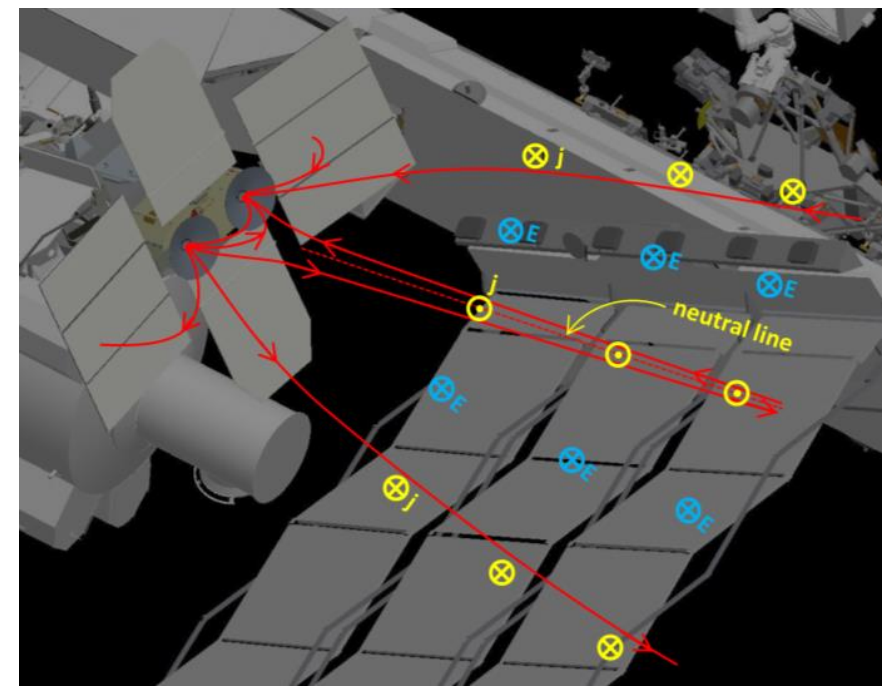

Figure 6. A cartoon of the magnetic field lines of the cancelling dipoles of the two rocket cores of the VF-200-1 in its active configuration with the motors firing. The plasma exhaust will create a helmet-streamer-like configuration. of $0.5 \mathrm{~mm}$. The red solid line in Figure $7 \mathrm{a}$ depicts the full axial range of possible plasma measurements. The red line extends into the VASIMR ${ }^{\circledR}$ VX-200 device, but does not penetrate the helicon source itself, and extends $5 \mathrm{~m}$ downstream into the expanding exhaust region of the vacuum chamber.

a)
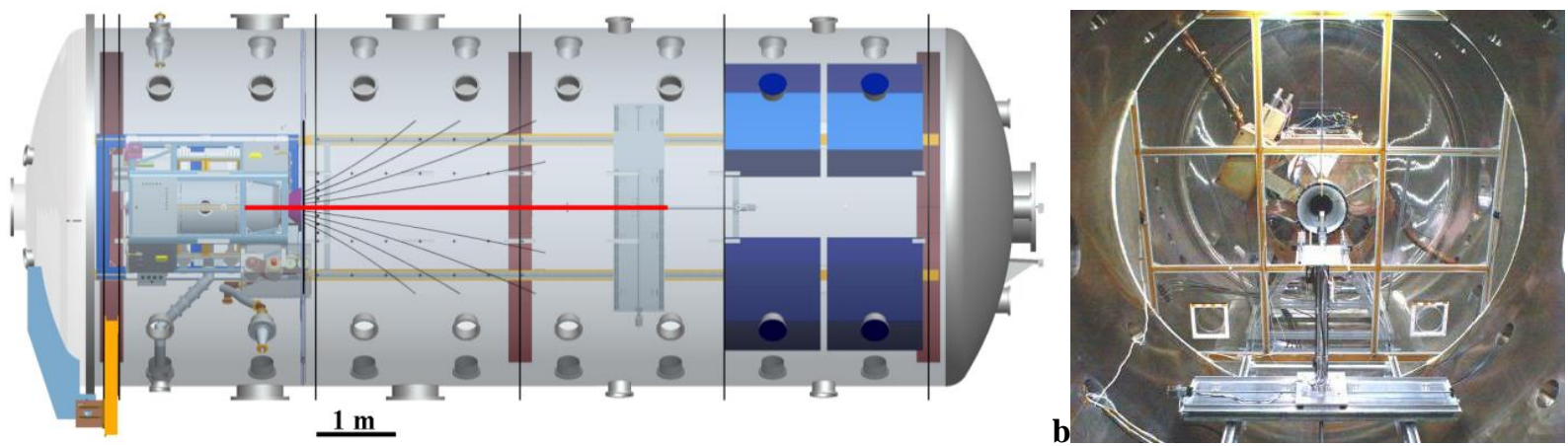

Figure 7. A CAD rendering of the VX-200 rocket bus mounted within the $150 \mathrm{~m}^{3}$ Ad Astra Rocket Company high vacuum facility with superimposed vacuum magnetic field lines (a), and a photograph of the VX-200 rocket (background) and diagnostics platform (foreground) mounted on a $2 \mathrm{~m}$ by 5 m translation stage (b).

AARC has auxiliary water cooled electromagnets and possible additional plasma sources that could enable us to simulate some aspects of the non-axisymmetric quadrupolar field geometry of the VF-200 in the laboratory. The 
magnets of an older VASIMR ${ }^{\circledR}$ model, the VX-100, have been refurbished, which mount external to main chamber on the right downstream of the VX-200 nozzle (Figure 8). The auxiliary electromagnets are approximately the same size and dimensions as the VF-200 superconductor, though there is an additional 1.0 meter diameter coil at the chamber port that could be used for magnetic geometry control.

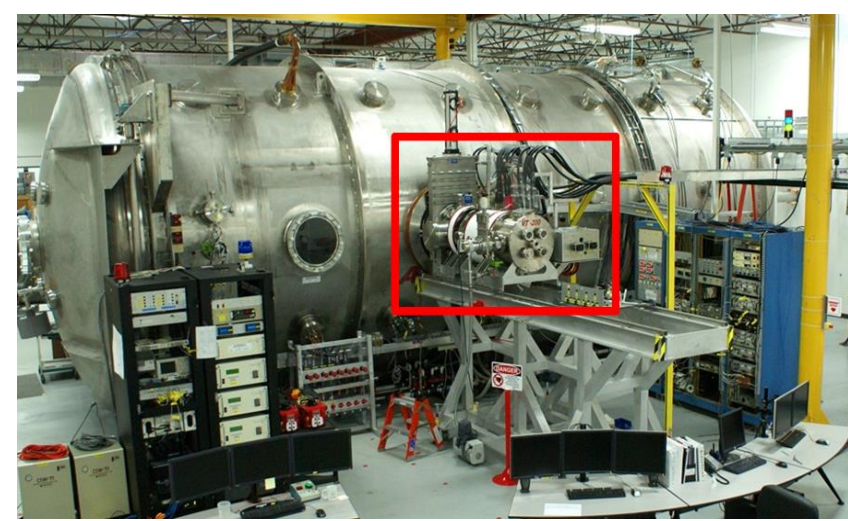

Figure 8. Photograph of the Ad Astra Rocket Company facility showing the auxiliary electromagnets (in red box). The electronics racks at the left are $\sim 2 \mathrm{~m}$ tall.
Developing the ISS Space Plasma Laboratory

Aurora is slated for installation on the ISS in 2015. To exploit the opportunity for in situ space plasma investigations using Aurora as a plasma source, it is vital now to begin developing the appropriate instrumentation suite and the necessary understanding of the anticipated plasma conditions in the exhaust. These are the objectives of our project. In particular, we will:

1. Plan the required instrumentation suite, acquire a prototype version of it, and employ it in terrestrial laboratory experiments with the existing VASIMR ${ }^{\circledR}$ VX-200. This work will be performed at the University of Houston (UH), the University of Michigan, and Ad Astra Rocket Company (AARC), using AARC's high-

capacity vacuum chamber.

2. Develop and validate a robust understanding of the VASIMR® plasma exhaust using sophisticated particle/kinetic and magnetofluid simulation models. These tasks will be performed at AARC and at NASA Goddard Space Flight Center (GSFC), respectively.

3. Integrate the laboratory experiments and numerical modeling to project the performance of the ISS VF-200 plasma source and its utility for in situ space plasma studies relevant to solar and heliospheric science. This work to achieve closure between the modeling, laboratory experiments, and solar/heliospheric observations will be performed at GSFC.

4. Finalize a plan and the associated costs for an instrumentation package that can be deployed on the ISS and describe the science that can be done with it. This report will conclude our concept study for the ISPL, and will involve UH, AARC, NRL, and GSFC.

With the ISS VF-200 we will perform pioneering space-plasma experimental science that has never been done before and will never be done in any terrestrial laboratory. The physical system that we will study, an open-closed dipolar magnetic configuration with reconnection in an extended current sheet, is the basis for major Heliophysics activity such as helmet streamers, eruptive flares, and magnetotail activity. The ISS VF-200 facility affords us a truly unique opportunity to make revolutionary advances in Heliophysics understanding.

\section{A. Subsatellite Instrumentation}

Detailed study of the magnetic reconnection processes taking place in the exhaust streamer of the VF-200 will require sophisticated in situ instrumentation. The investigations will require deployment of a platform that can penetrate the exhaust streamer at distances up to $25 \mathrm{~m}$ downstream. Many of the envisaged instruments will need to operate at or above the plasma potential in the exhaust; since this potential may be tens of volts different from the ISS spacecraft ground, the in situ instruments need to be deployed on an electrically isolated package. This requirement can best be addressed by the use of a Remote Manipulator System (RMS) subsatellite. The subsatellite concept named the Aurora Plasma Diagnostics Package (APDP) is shown deployed on the RMS in Figure 9. 
The concept of using a Plasma Diagnostics Package (PDP) subsatellite to investigate high power plasma sources in space was developed as part of the Shuttle/Spacelab system ${ }^{20,21}$. The PDP flew twice, on STS-3 in conjunction with the OSS-1 experiment pallet ${ }^{22,23,24,25}$ and on STS-51F, in conjunction with Spacelab 26,27,28. The PDP instrumentation included a Langmuir probe, density fluctuation, electric, and magnetic field wave receivers, a neutral pressure gauge, low energy electron and proton electrostatic analyzers, a ion mass spectrometer, a retarding potential analyzer (RPA), a differential ion flux probe, dc electric field and magnetometer, and a Faraday cup ${ }^{21,22}$. The PDP was a cylinder $1.07 \mathrm{~m}$ diameter by $0.69 \mathrm{~m}$ height (since the proposed APDP will not be a free flyer, it will be smaller) that weighed $159 \mathrm{~kg}$ and used $45 \mathrm{~W}$ of power. The scientific results obtained by the PDP are too extensive to repeat in detail here. The results of greatest relevance were observations of plasma wave generation by the energetic electron beam from the Fast Pulsed Electron Gun experiment on STS- $3^{22}$; of plasma wave generation by the orbiter itself ${ }^{26}$; and of changes in the ion distribution function in the orbiter wake ${ }^{28}$. A major effort of the work described here will be to refine the instrument selection for the APDP. The PDP instrument suite contained many of the types of instruments that will be used to study the VF-200 exhaust streamer. A major part of our planned work effort will be to refine the selection, add remote sensing instruments, and estimate the required gains, sensitivities, dynamic ranges and geometric factors. The key lesson of our experience with the PDP experiments is the demonstration that it is possible to use a subsatellite package on the RMS arm to make in situ observations of high-energy plasma experiments safely. The 15-m length of the RMS and the $10 \mathrm{~m}$ available traversal range of the RMS mounting cart downstream of the VF-200 nozzles will open an estimated $25 \mathrm{~m}$ length of the exhaust streamer to direct investigation. All of the low-energy plasma probes will be forward mounted on booms of $1 \mathrm{~cm}$ diameter and 1-2 m length to reduce interference with the exhaust plume by the notional $65 \mathrm{~cm} \times 50 \mathrm{~cm} \times 50 \mathrm{~cm}$ package body. The APDP will intersect $\sim 2 \%$ of the plume area at closest approach and $\sim 0.3 \%$ at farthest approach.

The plasma exhaust plume features will have scale lengths on the order of 0.05 to $0.1 \mathrm{~m}$. The APDP package will be designed to withstand the plasma bombardment and heat flux from 0.5 to $25 \mathrm{~m}$ from the VF-200 exhaust. This large range of motion will permit observations of axial and radial ambipolar electric field structures ${ }^{32}$ previously $^{2}$ observed in the lab, in addition to farther out regions where the plasma pressure dominates. Reconnection can occur in the far plume where the plasma pressure dominates everywhere, but solar-relevant reconnection will occur where the magnetic pressure dominates on either side of the current sheet where the field reverses.

\section{B. Modeling}

In order to plan the components and positioning of the APDP instrumentation package for optimal scientific return from the ISS experiments, it is vital to understand the anticipated magnetic-field and plasma characteristics of the engine exhaust streamer across Aurora's expected range of operational parameters. Important insights into these requirements already are available from laboratory experiments conducted on Aurora's predecessor, the VX-200 single-core, magnetic-dipole engine, together with the specifications for the VF-200 facility. However, the ISS configuration will consist of two rocket cores connected magnetically to each other, embedded in a quadrupolar magnetic geometry, and surrounded by the vacuum of space without confining metallic walls. The effects of these new features on the behavior of the Aurora exhaust are poorly understood and demand quantitative investigation by detailed models validated by laboratory experiments. The development of the VASIMR ${ }^{\circledR}$ plasma engine has seen a substantial and fruitful interplay between analytical and numerical models on the one hand and laboratory prototype experiments on the other ${ }^{29}$. Topics addressed in these investigations include single-pass ion-cyclotron resonance heating in the rocket core ${ }^{16,30}$, ambipolar ion acceleration in the nozzle $^{31,32}$, and trans-Alfvénic plasma detachment in the expanding magnetic field of the near-nozzle region ${ }^{18,33,34}$. This successful modeling research now must be extended to address $3 \mathrm{D}$ structures in the magnetoplasma, time-dependent phenomena in the system, and the character and evolution of the

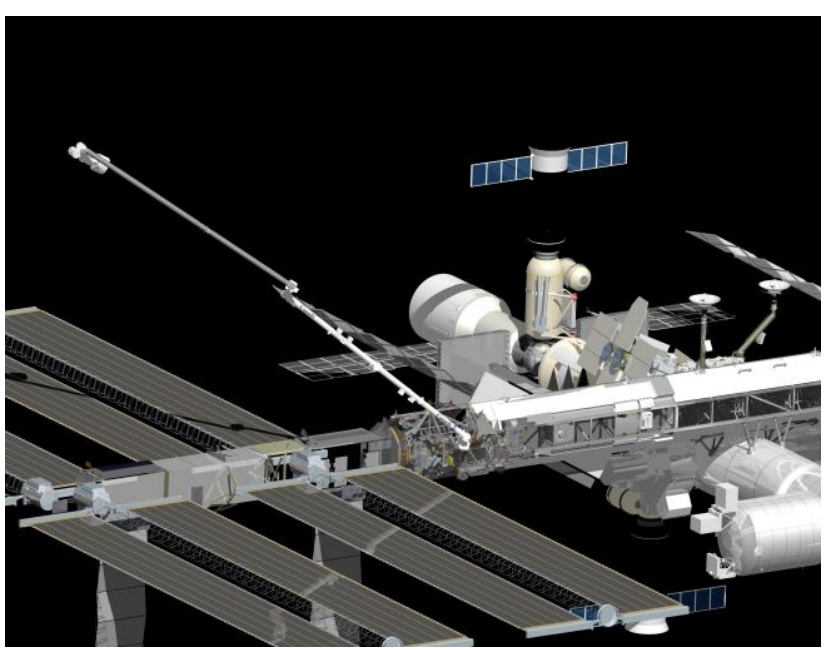

Figure 9. Artist's conception of the APDP mounted on the RMS arm and an extension boom in position to observe the VF-200 exhaust plume. The exhaust plasma is not shown. 
To acquire the needed quantitative information and qualitative insight, we will use the Adaptively Refined Magnetohydrodynamics Solver (ARMS). ARMS solves the time-dependent, nonlinear equations of MHD, using fully 3D Flux-Corrected Transport convection algorithms ${ }^{35}$ that are designed specifically to treat robustly shock waves, current sheets, and other MHD discontinuities. ARMS also employs adaptive mesh refinement and massively parallel computing via GSFC's PARAMESH toolkit $^{36}$, so that unprecedented spatial resolution can be concentrated precisely where it is needed. ARMS has been used to investigate reconnectionrelated phenomena in the solar corona in a number of contexts and at unprecedented numerical resolution $^{37,38,39,40,41,42,43,44,45,46}$.

The exhaust streamer plume of the VASIMR ${ }^{\circledR}$ engine and helmet streamers in the solar wind are physically exact analogues, as indicated in Figures 6 and 10. Both originate in regions - the rocket core and the low corona - where the plasma thermal $(P)$ and kinetic $\left(\rho v^{2} / 2\right)$ pressures are much smaller than the magnetic pressure $\left(B^{2} / 8 \pi\right)$. With increasing distance from those sources, however, the magnetic pressure declines more rapidly than the plasma pressures due to the divergent field geometry of the rocket nozzle and the outer corona, respectively. The plasma thermal energy drives an expansion that eventually becomes both supersonic and super-Alfvénic, and produces a magnetic configuration consisting of open and closed field exactly as a coronal streamer.

We will perform numerical simulations with ARMS to understand of the 3D structure and time dependence of the VASIMR ${ }^{\circledR}$ plasma engine's exhaust. Existing models already replicate the behavior of the rocket core proper. Thus, the simulation domain for ARMS will begin at the nozzle outlet, which will be a small part of the left $x$ wall of a Cartesian domain, with the engine axis oriented along the $+x$ direction. The right $x$ wall and the four side walls in $y$ and $z$ will be placed according to the simulated scenario: at the distances prescribed by the AARC vacuum chamber dimensions and with closed boundaries to study the laboratory VX-200 and VT-200; far away and with open boundaries to emulate the ISS space environment of the VF-200-1. The initially uniform plasma and vacuum magnetic field will be perturbed by the inflowing plasma specified at the left $x$ wall, as it propagates into and across the domain, stretching out the magnetic flow tube on which it resides.

Our initial scoping studies will be performed with ARMS in its one-fluid, one-temperature, ideal-MHD mode with isotropic thermal pressure. All that needs to be added are implementations of the initial vacuum magnetic field and of the circular nozzle inlet region(s) at the wall, for which AARC will provide specifications. These baseline numerical experiments will be done first, to provide both a first rough check against existing VX-200 experiments and a starting point for predicting exhaust-streamer conditions and needed diagnostics for the ISPL's VF-200-1.

Subsequent efforts will be devoted to refining the ARMS model and conducting more detailed comparisons between the numerical and laboratory experiments on the simple VX-200 and the more complex VT-200 with its orthogonal second core and magnetic field. We will include field-aligned electron thermal conduction and determine its significance in assisting the thermal expansion. We also will generalize our MHD model to permit the electron and ion temperatures to differ, and furthermore to allow for distinct ion temperatures (and pressures) parallel and perpendicular to the magnetic field. This step will require the straightforward inclusion of an ion pressure tensor and two new adiabatic equations for $T_{\|}$and $T_{\perp}{ }^{47}$. This addition is motivated by measurements of the semi-collisional VX-200 plasma showing that conditions of unequal, anisotropic temperatures occur in the VASIMR nozzle region ${ }^{16}$ and should persist in the exhaust. In that case, the far exhaust streamer may be subject to a variety of instabilities thought to be important in the solar wind ${ }^{48}$, such as the MHD parallel firehose instability ${ }^{49}$ that excites large-amplitude Alfvén waves.

This extended and improved ARMS model, validated against the proposed laboratory experiments, will be employed over the course of the program to project the expected conditions in the Aurora plume of the ISPL. Aurora 
will consist of two identical VX-200 cores arrayed side-by-side with antiparallel magnetic dipole fields and no walls to confine the exhaust plume. This configuration is actually physically simpler than the laboratory setups, which pose the greater modeling challenge.

\section{Experiment Design Process}

Full power operation of the VF-200 will produce a helmet-streamer-like configuration of highly stretched antiparallel field lines with a Y-type current sheet separating regions of opposite polarity. In this configuration, the active reconnection sites are expected to occur downstream at distances that are a few multiples of the nozzle separation $(1.5 \mathrm{~m})$ of the VF-200, consistent with the geometry of helmet streamers and also with the position of reconnection sites in the terrestrial magnetotail. These locations will be well within the range $(0-25 \mathrm{~m})$ of the RMS arm deploying the APDP. It will be most fruitful to design specific VF-200 operating modes that force driven reconnection to occur at predictable locations where the APDP can be placed. The VF-200 control parameters available for adjustment include ICH power level, which can be varied at frequencies up to $20 \mathrm{kHz}$; gas flow, which can be varied on time scales down to milliseconds; and the balance of gas flow and ICH power between cores. One of the objectives of the ARMS modeling described above will be to identify both the operating modes and the input parameter time profiles that will force reconnection to occur at a predicted place.

\section{Instrument Selection and Design}

The basic physics of the processes that will be studied defines the instrument selection pretty well. The instrument complement of the original PDP is a good starting point. The initial strawman design adopted for the purpose of further study omits the dc electric field detectors and adds cameras, including broadband visible light and Ar II emission line imagers. The results of the initial modeling effort will provide the input needed to refine this selection and choose gains, geometric factors, etc. The instruments will be prioritized by the Science Working Team based on their importance to specific science objectives.

The team will also review the overall instrument selection to evaluate if any key possibilities have been overlooked. Candidates for inclusion include a total electron content monitor, a dc electric field detector, an array of multiple small search coils, other imagers and x-ray and energetic particle detectors. Particular attention will be paid to looking for evidence in the models that the reconnection region might be sufficiently active to accelerate electrons to 10 's of $\mathrm{keV}$ or higher.

The strawman instrument complement of the APDP is generally so well-known and published that detailed descriptions are superfluous, particularly since the details of the required sensitivities are not known. Since the VF-200 is going to be substantially denser and faster flowing than normal ionospheric

Table 1 APDP Instrument List

Definite

Dc triaxial fluxgate magnetometer

Single and double Langmuir probe

Retarding Potential analyzers

Faraday cup

Ion mass spectrometer

Pressure gage

Argon and electron differential energy analyzer

Ac magnetic wave search coil sensor

Ac electrostatic wave analyzer

Broadband visible light imager

Ar II emission line imager

\section{Candidates}

Total electron content

Dc electric field detector

Array of small search coils

Energetic particle detectors

$\mathrm{X}$-ray imager plasma, it should be clear that signal to noise ratios and adequate minimum sensitivity will not be issues. However, the non-standard parameter range means that many instruments that could be build-to-print or spares from a previous program will require more careful attention.

Thermal management design will be the biggest design challenge of the prototyping process. Close in to the nozzle of the VX-200, the exhaust has proven more than adequately energetic to melt probes and require use of highly refractory materials. Ordinary ionospheric instruments never encounter conditions similar to those that will be found in the VF-200 exhaust. The basic outline of the planned design process will be to design the sensor heads of each instrument to function in the expected thermal environment. The APDP subsatellite bus will be designed with an insulating barrier between the rocket exhaust and the instrument data transducer and data handling electronics, along with the spacecraft systems.

Once the instrument complement has been selected, the models will be used to estimate the signal ranges that each instrument will be required to detect to address the science objectives. Sensor dimensions and amplifier gains will then be chosen to provide adequate sensitivity and signal to noise to make the needed measurements with the required precision. 


\section{E. Data Analysis}

\section{Computational and Modeling Resources}

All UH data reduction and analysis software will be written in Interactive Data Language (IDL), which is powerful and easily ported to different operating systems. Each general public user will be able to download any version of the UH software package, either entirely or one task at a time, from a web page maintained at UH. All project software will be version controlled and quality checked by the University of Houston. The existing UH CVS repository for MINIS software will be used for version control on this project. The UH MINIS CVS repository of IDL routines is located on the UH space physics process server, space.phys.uh.edu. All approved project software developers will have password protected access to the repository via the Secure Shell (SSH2) protocol. Standard CVS check out/check in procedures will be used for version control, with UH staff having final review and approval.

The ARMS numerical simulations required for this project will be conducted and archived on DoD and NASA computational resources. Printed and graphical outputs, including images from the HelioSpace visualization tool (cf. Figure 10), also will be transferred to the UH computer system for team and public access.

2. In-depth Analysis

The analysis plan is fairly standard in structure. This plan will only be used if we complete the prototype in time to begin initial testing within the 3-year period of this investigation. In outline, the process consists of data reduction and validation; organizing, collating and plotting data from multi-shot parameter scans; modeling comparisons and validation; and detailed interpretation. Each objective will be assigned to a specific team member to be analysis lead.

In the first step, software suites that are controlled by modular graphical user interfaces will be used retrieve the raw data, apply calibrations and instrument response models to produce data vectors in physical units, which will be written back out to analyzed data nodes in the MDSplus tree. UH data reduction software is written in IDL, while the Ad Astra suite is maintained in MATLAB. The team has sufficient expertise in both languages to manage and develop this combined language suite successfully. In the second step, data from multiple shots are combined to explore extended parameter spaces. This task will be carried out using custom IDL software already developed by the UH team.

The final steps of model comparison and interpretation will involve comparing data with predictions of various reconnection and exhaust-streamer models. There are several models available to this team that have not yet been run using combined VX-200 and VT-200 initial and boundary conditions. If necessary, these models may be extended and run as part of this work in addition to the ARMS model described above. These models include the University of Texas MHD magnetic nozzle code ${ }^{31,33}$, Ad Astra PT, a particle trajectory code for plasma exhaust modeling ${ }^{50}$ [Ilin et al., 2004], and AARC Transport3d, a Boltzmann electron model with ion trajectories that uses 3$\mathrm{d}$ coil configurations. Depending on the outcome of the comparisons between the experimental results and the ARMS simulations, we will run these models to target specific physics questions.

The results of both the laboratory and numerical simulations will also be compared with observational data from several missions; in particular SDO and the coronagraphs on SOHO and STEREO, to find evidence of the processes on the Sun that correspond to the models. We will perform an extensi on the SOlar and Heliospheric Observatory (SOHO) and the Solar TErrestrial RElations Observatory (STEREO), exploration of available solar data and compile a comprehensive data set of the observational evidence for the type of reconnection expected from the experiments. This data set will also be essential for the future IPSL studies.

\section{F. Data Archiving and Dissemination}

The raw laboratory data are stored by the instrumentation digitization system in an MDSplus server at Ad Astra Rocket Company. MDSplus is community based open source software developed for the magnetic fusion energy program by the Massachusetts Institute of Technology, the Fusion Research Group in Padua, Italy, and the Los Alamos National Laboratory. It is the most widely used system for data management in the laboratory plasma physics community, and is therefore suitable for use as a public dissemination tool. MDSplus is a set of software tools for data acquisition, storage and management. MDSplus stores data in a single, self-descriptive hierarchical tree. The tools permit easy expansion of tree models to include a wide variety of data sources, including analysis data products. Since it uses a client/server model, it is well suited to providing public and remote access to the data.

The University of Houston (UH) will comply with all requirements to provide public access to project data. All project laboratory data that does not contain either ITAR controlled or proprietary information will be stored in realtime in a separate sub-tree of each shot on the main MDSplus data base. Copies of this subtree from all project related VASIMR ${ }^{\circledR}$ shots will be placed on a separate MDSplus server located at Ad Astra Rocket Company building in a server room physically outside the ITAR controlled area and logically outside the firewall. Interested community users will able to register and obtain a password to the MDSplus archive. 


\section{G. Connection to the Sun/Heliosphere}

Whereas the magnetic topology of the VF-200 exhaust is identical to that of a helmet streamer, the global spatial scales obviously are not; therefore, a critical aspect of our research program will be to determine how best to relate the experimental results to the Sun. Although the global scales are very different, the actual plasma parameters and kinetic scales are not far from coronal values. The plasma beta (ratio of thermal to magnetic pressure) and Mach number of the VF-200 exhaust are comparable to those in helmet streamers and the inner solar wind. The noble-gas propellants of the engine (argon in typical operating mode; neon and helium will be examined for use in ISPL experiments) are predominantly singly ionized, more massive than the protons that are the main constituent of the solar wind, but providing a simpler one-species plasma for investigating the reconnection physics in the plume.

Table 2 lists several key parameters for comparing the ISPL plume to the solar corona. We assume a coronal scale length $(100 \mathrm{~m})$ that has the same travel time for sonic flow $(1 \mathrm{~ms})$ as that of the VASIMR ${ }^{\circledR}$ exhaust plume traversing the APDP scale size $(10 \mathrm{~m})$. To be sure, this is but a small section of a far larger, extended coronal current sheet. The section sampled by the ISPL, however, encompasses all important electron and ion kinetic scales. Thus, experiments performed on the ISS will be able to investigate the flux-breaking processes occurring inside coronal current sheets. The only discrepancy between ISPL experiments and the corona is that the currently cold VASIMR ${ }^{\circledR}$ electrons have mean free paths comparable to, rather than much larger than, the APDP scale.

As the table shows, the electron skin depth and gyro-radius are both very small throughout the VF-200 exhaust; meanwhile, the ion skin depth and gyroradius are about $4 \mathrm{~m}$ and $0.7 \mathrm{~m}$, respectively, quite similar to those in the corona. These ion lengths are sufficiently large that the current sheet can be probed experimentally without difficulty, enabling examination of sheet properties at the kinetic scales where magnetic-flux breaking occurs in reconnection. At the same time, the lengths are small enough that the current sheet can extend to hundreds of skin depths, while remaining within the $25-\mathrm{m}$ reach of the moveable arm supporting the APDP. This is the great advantage of the open ISPL configuration for reconnection studies: a large currentsheet aspect ratio is absolutely essential for relating the laboratory experiments to the solar/heliospheric case, which is expected to have very high aspect ratios. We anticipate achieving Lundquist numbers $S$ in the ISPL plume that are at least on the order of $10^{4}$, much larger than in other laboratory experiments, and comparable to current high-resolution, 3D numerical simulations of reconnecting current sheets $^{43,51,52,53}$. The recent studies predict a transition at $S \sim 10^{4}$ from slow SweetParker reconnection to fast reconnection dominated by formation of plasmoids, whose number scales with $S$. If correct, this result has enormous implications for understanding explosive Heliophysics activity. Laboratory studies clearly are needed to provide experimental tests of these predictions; those studies in turn will increase our confidence in extrapolating the numerical models toward the solar regime of $S \sim 10^{12}$.

\begin{tabular}{|cccc|}
\hline Table 2 & \multicolumn{4}{l}{ ISPL/Corona Parameters } & \\
Quantity & Symbol (Units) & $I S P L$ & Corona \\
Number density & $n\left(\mathrm{~m}^{-3}\right)$ & $5 \times 10^{15}$ & $1 \times 10^{14}$ \\
Electron temperature & $T_{\mathrm{e}}(\mathrm{K})$ & $1 \times 10^{4}$ & $1 \times 10^{6}$ \\
Ion temperature & $T_{\mathrm{i}}(\mathrm{K})$ & $3 \times 10^{5}$ & $1 \times 10^{6}$ \\
Magnetic field & $B(\mathrm{G})$ & 10 & 10 \\
Ion mass number & $m_{\mathrm{i}} / m_{\mathrm{p}}$ & $2(\mathrm{He})$ & $1(\mathrm{H})$ \\
Flow velocity & $V\left(\mathrm{~km} \mathrm{~s}^{-1}\right)$ & 10 & 100 \\
Sound speed & $C_{\mathrm{S}}\left(\mathrm{km} \mathrm{s}^{-1}\right)$ & 10 & 100 \\
Alfvén speed & $\left.V_{\mathrm{A}}(\mathrm{km} \mathrm{s})^{-1}\right)$ & 200 & 2000 \\
System length & $L(\mathrm{~m})$ & 10 & 100 \\
Transit time & $\tau(\mathrm{ms})$ & 1 & 1 \\
Ion plasma frequency & $\omega_{\mathrm{pi}} \tau$ & $7 \times 10^{+4}$ & $1 \times 10^{+4}$ \\
Ion cyclotron frequency & $\omega_{\mathrm{ci}} \tau$ & $5 \times 10^{+1}$ & $1 \times 10^{+2}$ \\
Ion/ion collision frequency & $v_{\mathrm{ii}} \tau$ & $1 \times 10^{-2}$ & $1 \times 10^{-4}$ \\
Electron plasma frequency & $\omega_{\mathrm{pe}} \tau$ & $4 \times 10^{+6}$ & $6 \times 10^{+5}$ \\
Electron cyclotron frequency & $\omega_{\mathrm{ce}} \tau$ & $2 \times 10^{+5}$ & $2 \times 10^{+5}$ \\
Electron/ion collision frequency & $v_{\mathrm{ei}} \tau$ & $2 \times 10^{+2}$ & $7 \times 10^{-3}$ \\
Ion skin depth & $d_{\mathrm{i}} / L$ & $4 \times 10^{-1}$ & $2 \times 10^{-1}$ \\
Ion gyroradius & $\rho_{\mathrm{i}} / L$ & $7 \times 10^{-2}$ & $1 \times 10^{-2}$ \\
Ion mean free path & $\lambda_{\mathrm{ii}} / L$ & $2 \times 10^{+2}$ & $8 \times 10^{+3}$ \\
Electron skin depth & $d_{\mathrm{e}} / L$ & $8 \times 10^{-3}$ & $5 \times 10^{-3}$ \\
Electron gyroradius & $\rho_{\mathrm{e}} / L$ & $2 \times 10^{-4}$ & $2 \times 10^{-4}$ \\
Electron mean free path & $\lambda_{\mathrm{e} i} / L$ & $2 \times 10^{-1}$ & $5 \times 10^{+3}$ \\
Debye length & $\lambda_{\mathrm{D}} / L$ & $1 \times 10^{-5}$ & $7 \times 10^{-5}$ \\
Plasma beta & $\beta$ & $5 \times 10^{-2}$ & $7 \times 10^{-3}$ \\
Mach number & $M$ & 1 & 1 \\
Alfvén number & $A$ & $5 \times 10^{-2}$ & $5 \times 10^{-2}$ \\
\hline
\end{tabular}


Our experiments certainly will not reach solar Lundquist numbers. No experiment or simulation can do that, because the physical systems that we construct - whether plasma engines or numerical grids - simply cannot get close to the spatial scales available to the Sun. In one critically important aspect, however, the ISPL experiment does fully capture reality: for the first time, the outer boundary of the system will be at infinity, just as it is in the Sun and the Heliosphere.

\section{H. Comparison with Solar/Heliospheric Observations}

Numerous studies have reported the dynamics of plasma density enhancements and flows issuing from the tops of helmet streamers, as observed remotely using the SOHO/LASCO ${ }^{2,54,55,56,57}$ and STEREO/SECCHI ${ }^{5,59}$ coronagraphs and STEREO/HI heliospheric imager $^{60}$, and measured in situ using plasma and magnetic-field instruments on STEREO, ACE, and Wind ${ }^{61,62}$. It is generally accepted that these relatively small-scale, slow-moving plasma blobs and their entrained flux ropes are released by intermittent magnetic reconnection events occurring near the streamer apices. The Y-type null points at the streamer tops have nearly static, closed field lines below the inverted Y, but open field lines with outflowing solar-wind plasma to both sides. Therefore, the null-point structure is constantly jostled, giving rise to a recurrent stretching out, pinching off, and relaxing downward of the outermost closed magnetic flux surfaces, with the concomitant outward ejection of a disconnected plasmoid between the innermost open flux surfaces. This phenomenon is illustrated by the image from our ARMS simulation shown previously in Figure 10, in which a newly disconnected flux rope with enhanced plasma density is slowly departing from the top of the helmet streamer.

Experimental investigation of the streamer-top reconnection process requires a facility such as the ISPL, in which the plasma can expand freely and stretch the magnetic field out to great distances. With it, we can address many questions about the governing reconnection mechanism. How do the properties (density, width, length, velocity, temperature, frequency) of the disconnected blobs depend upon the parameters of the streamer? Are energetic particles accelerated by the reconnection electric fields? Is the reconnection steady or intermittent? What governs its temporal behavior? Does resistive MHD provide an adequate representation of the reconnection dynamics? Is the resistivity Spitzer-like (determined by particle/particle collisions), anomalous (set by microscopic plasma turbulence), or kinetic (fixed by flux breaking at the ion skin depth)? Although the ISPL will not reach the true coronal regime of extremely high Lundquist numbers, it will attain very large values that are within our capacity to simulate numerically and that will pose unprecedented tests of our current physical understanding. By determining the scaling of the experimental and numerical results that are within reach, we will extrapolate our findings toward the realm of coronal parameters, for comparison with available and future remote sensing and in situ observations of helmet-streamer dynamics and the slow solar wind. The resulting advances in our understanding of magnetic reconnection in space plasmas will have far broader impact on Heliophysics, however, ranging from the physics of eruptive flares and CMEs at the Sun to magnetopause and magnetotail reconnection processes at Earth.

\section{Conclusion}

This project is primarily an instrument development project that will produce a Concept Study report and prototype instrumentation. Real science closure on the ultimate goals can only be obtained by actual flight of the APDP mission and experiments on the ISS. Those future ISPL experiments will enable us to attack the fundamental science question that we posed:

- What is the role of reconnection in opening and closing the solar magnetic field?

The uniquely wall-less ISPL will support in situ experiments with open magnetic fields that cannot be performed in any terrestrial laboratory. The dynamic interplay at the boundary between open and closed fields, which may well be the origin of the slow solar wind ${ }^{5}$, will be directly observable with the APDP instrumentation on the ISS. More generally, the fundamental process of reconnection can be studied in a true space environment with open magnetic fields, whose location and rate can be controlled by changing the operating parameters of the VF-200 plasma engine. Measurements of electric and magnetic fields and of particle distribution functions in the exhaust streamer will enable us to answer important, long-standing questions about the magnetic-reconnection process:

- What determines the rate of reconnection, and whether or not it is bursty?

- How is the released energy partitioned between thermal, kinetic, and particle?

The work performed under this project will develop the instrumentation package for the ISPL, validate models for predicting the behavior of the ISPL exhaust streamer by comparing their predictions with data on simpler laboratory configurations, and predict specific modes of operation of the ISPL that will be most fruitful for improving our understanding of reconnection. We also will begin to compile a list of solar events, observed at high 
resolution and high cadence by SDO/AIA, that seem to be promising candidates for comparison with the VF-200 exhaust-streamer simulations and with eventual ISPL experiments. These essential steps lay the foundation for an ISPL that will provide revolutionary advances in our knowledge of solar and heliospheric physics.

\section{Acknowledgments}

Benjamin Longmier would like to thank the University of Houston Institute for Space Systems Operations (ISSO) postdoctoral fellowship program for partial support of this research. NASA Johnson Space Center under grant NAG 9-1524, the Texas Higher Education Coordinating Board under Advanced Technology Program project 003652-0464-1999 and the Ad Astra Rocket Company sponsored this research.

\section{References}

${ }^{1}$ Schwenn, R., et al., First view of the extended green-line emission corona at solar activity minimum using the LASCO-C1 coronagraph on SOHO, Sol. Phys., 175, 667, 1997.

${ }^{2}$ Sheeley, N. R., Jr., H. P. Warren, and Y.-M. Wang, A streamer ejection with reconnection close to the Sun, Ap.J., 671, 926, 2007.

${ }^{3}$ Kunow, H., N. Crooker, J. Linker, R. Schwenn, and R. von Steiger, Coronal Mass Ejections, Space Sciences Series of ISSI, Vol. 21 (Bern: ISSI), 2007.

${ }^{4}$ Antiochos, S. K., C. R. DeVore, J. T. Karpen, and Z. Mikić, Structure and dynamics of the Sun's open magnetic field, Ap.J., 671, 936 doi: 10.1086/522489, 2007.

${ }^{5}$ Antiochos, S. K., Z. Mikić, R. Lionello, V. S. Titov, and J. A. Linker, A model for the sources of the slow solar wind, Ap.J., $731,112,2011$.

${ }^{6}$ Sheeley, N. R., Jr., H. P. Warren, and Y.-M. Wang, Ap.J., 616, 1224, 2004.

${ }^{7}$ Zweibel, E. G., and M. Yamada, Magnetic reconnection in astrophysical and laboratory plasmas, Ann. Rev. Astron. Astrophys., 47, 291-332, 2009.

${ }^{8}$ Yamada, M., R. Kulsrud, and H. Ji, Magnetic reconnection, Rev. Mod. Phys., 82, 603-665, doi: 10.1103/RevModPhys.82.603, 2010.

${ }^{9}$ Chang Díaz, F. R., J. P. Squire, A. V. Ilin, A. J. Petro, R. D. Bengtson, B. N. Breizman, F. W. Baity, M. D. Carter, R. Z. Sagdeev, K. Molvig, E. A. Bering, A. A. Chan, and D. Micheletti, An overview of current research on the VASIMR engine. Paper presented at the 2000 APS-DPP Mini Conference on Plasma Propulsion, 42nd Annual Meeting of the APS Division of Plasma Physics, Quebec City, Canada, October 23-27, 2000.

${ }^{10}$ Chang Díaz, F. R., J. P. Squire, E. A. Bering III, J. A. George, A. V. Ilin, A. J. Petro, and L. Cassady, The VASIMR engine approach to solar system exploration. Paper presented at the 39th AIAA aerospace sciences meeting and exhibit, Reno, NV, Jan 8-11, 2001.

${ }^{11}$ Chang Díaz F. R., J. P. Squire, A. V. Ilin, A. J. Petro, V. Jacobson, G. McCaskill, A. G. Tarditi, D. A. Winter, R. D. Bengtson, B. N. Breizman, F. W. Baity, M. D. Carter, P. Colestock, M. Light, E. A. Bering, T. W. Glover, C. Davis, and D. G. Chavers, Early Results of ICRH Experiments in VX-10, Bulletin of the American Physical Society, DPP03, RP1.138, RP1.138, 2003.

${ }^{12}$ Chang Díaz, F. R., J. P. Squire, T. Glover, A. J. Petro, E. A. Bering III, F. W. Baity Jr., R. H. Goulding, M. D. Carter, R. D. Bengtson, and B. N. Breizman, The VASIMR engine: Project status and recent accomplishments. Paper presented at the 42nd AIAA Aerospace Sciences Meeting and Exhibit, Reno, NV, January 5-8, 2004.

${ }^{13}$ Bering, E. A. III, F.R. Chang Díaz, J. P. Squire, The uses of RF waves in space propulsion systems, Radio Science Bulletin, 310, 92-106, 2004a. (Invited)

${ }^{14}$ Bering, E. A., III, F. R. Chang Díaz J. P. Squire, T. W. Glover, R. D. Bengtson, and M. Brukardt, Velocity phase space studies of ion dynamics in the VASIMR engine. Paper presented at the 42nd AIAA Aerospace Sciences Meeting and Exhibit, Reno, NV, January 5-8, 2004b.

${ }^{15}$ Bering, E. A., III, F. R. Chang Díaz, J. P. Squire, M. Brukardt, T. W. Glover, R. D. Bengtson, V. T. Jacobson, G. E. McCaskill, and L. Cassady, Electromagnetic ion cyclotron resonance heating in the VASIMR, Adv. Space Res., 42, 192-205, doi:10.1016/j.asr.2007.09.034, 2008.

${ }^{16}$ Bering, E. A., III, F. R. Chang Díaz, J. P. Squire, T. W. Glover, M. D. Carter, G. E. McCaskill, B. W. Longmier, M. S. Brukardt, W. J. Chancery, and V. T. Jacobson, Observations of single-pass ion cyclotron heating in a transsonic flowing plasma, Phys. Plasmas, 17, 043509, doi: 10.1063/1.3389205, 2010.

${ }^{17}$ Correll, D. L., H. Bohmer, N. Rynn, and R. A. Stern, Temporal evolution of ion temperatures in the presence of ion cyclotron instabilities, Phys. Fluids, 20, 822-828, 1977.

${ }^{18}$ Breizman, B. N., M. R. Tushentsov, and A. V. Arefiev, Magnetic nozzle and plasma detachment model for a steady-state flow, Phys. Plasmas, 15, 57103, 2008.

${ }^{19}$ Longmier, B. W., L. D. Cassady, M. G. Ballenger, M. D. Carter, F. R. Chang Diaz, T. W. Glover, A. V. Ilin, G. E. McCaskill, C. S. Olsen, and E. A. Bering III, VX-200 magnetoplasma thruster performance results exceeding 50\% efficiency, J. Prop. Power, 27, 915, $2011 \mathrm{~b}$. 
${ }^{20}$ Shawhan, S. D., Description of the plasma diagnostics package (PDP) for the OSS-1 shuttle mission and JSC chamber test in conjunction with the fast pulse electron gun (FPEG), in Artificial Particle Beams in Space Plasma Studies, edited by B. Grandal, pp. 419-430, Plenum, New York, 1982.

${ }^{21}$ Shawhan, S. D., J. L. Burch, and R. W. Fredericks, Subsatellite studies of wave, plasma and chemical injections from Spacelab, J. Space. Rockets, 20, 238-244, doi: 10.2514/3.25586, 1983.

${ }^{22}$ Shawhan, S. D., G. B. Murphy, P. M. Banks, P. R. Williamson, and W. J. Raitt, Wave emissions from dc and modulated electron beams on STS 3, Radio Sci., 2, 471-486, doi: 10.1029/RS019i002p00471, 1984a.

${ }^{23}$ Shawhan, S. D., G. B. Murphy, and D. L. Fortna, Measurements of electromagnetic interference on OV102 Columbia using the plasma diagnostics package, J. Space. Rockets, 21, 392-397, doi: 10.2514/3.25668, 1984b.

${ }^{24}$ Shawhan, S. D., G. B. Murphy, and J. S. Pickett, Plasma diagnostics package initial assesment of the Shuttle orbiter plasma environment, J. Space. Rockets, 21, 387-391, doi: 10.2514/3.25667, 1984c.

${ }^{25}$ Reasoner, D. L., S. D. Shawhan, and G. D. Murphy, Plasma diagnostics package measurements of ionospheric ions and shuttle-induced perturbations, J. Geophys. Res., 91, 13463-13471, 1986.

${ }^{26}$ Gurnett, D. A., W. S. Kurth, J. T. Steinberg, and S. D. Shawhan, Plasma wave turbulence aboard the Shuttle: Results from the Spacelab-2 flight, Geophys. Res. Lett., 15, 760-763, doi:10.1029/GL015i008p00776, 1988.

${ }^{27}$ Murphy, G. B., D. L. Reasoner, A. Tribble, N. D'Angelo, J. S. Pickett, and W. S. Kurth, The plasma wake of the Shuttle orbiter, J. Geophys. Res., 94, 6866-6873, 1989.

${ }^{28}$ Sorenson, J. E., N. H. Stone, and K. H. J. Wright, Change in ion distribution function while crossing the space shuttle wake, J. Geophys. Res., 102, 24117-24126, doi: 10.1029/97JA01869, 1997.

${ }^{29}$ Arefiev, A. V., and B. N. Breizman, Theoretical components of the VASIMR plasma propulsion concept, Phys. Plasmas, 11, 2942-2951, 2004.

${ }^{30}$ Breizman, B. N., and A. V. Arefiev, Single-pass ion cyclotron resonance absorption, Phys. Plasmas, 8, 907-915, 2001.

${ }^{31}$ Arefiev, A. V., and B. N. Breizman, Ambipolar acceleration of ions in a magnetic nozzle, Phys. Plasmas, 15, 42109, 2008.

${ }^{32}$ Longmier, B. W., E. A. Bering III, M. D. Carter, L. D. Cassady, W. J. Chancery, F. R. Chang Diaz, T. W. Glover, N. Hershkowitz, A. V. Ilin, G. E. McCaskill, C. S. Olsen, and J. P. Squire, Ambipolar ion acceleration in an expanding magnetic nozzle, Plasma Sources Sci. Technol., 20, 015007, doi: 10.1088/0963-0252/20/1/015007, 2011a.

33 Arefiev, A. V., and B. N. Breizman, Magnetohydrodynamic scenario of plasma detachment in a magnetic nozzle, Phys. Plasmas, 12, 043501-1-10, 2005.

${ }^{34}$ Deline, C. A., et al., Plume detachment from a magnetic nozzle, Phys. Plasmas, 16, 33502, 2009.

${ }^{35}$ DeVore, C. R., Flux corrected transport techniques for multidimensional compressible magnetohydrodynamics, J. Comput. Phys., 92, 142, 1991

36 MacNeice, P. J., K. M. Olson, C. Mobarry, R. de Fainchtein, and C. Packer, PARAMESH: A parallel adaptive mesh refinement community toolkit, Comput. Phys. Commun., 126, 330, 2000.

${ }^{37}$ DeVore, C. R., and S. K. Antiochos, Homologous confined filament eruptions via magnetic breakout, Ap.J., 680, $740,2008$.

${ }^{38}$ Lynch, B. J., S. K. Antiochos, C. R. DeVore, J. G. Luhmann, and T. H. Zurbuchen, Topological evolution of a fast magnetic breakout CME in three dimensions, Ap.J., 683, 1192, 2008.

${ }^{39}$ Lynch, B. J., S. K. Antiochos, Li, Y., J. G. Luhmann, and C. R. DeVore, Rotation of coronal mass ejections during eruption, Ap.J., 697, 1918, 2009.

${ }^{40}$ Pariat, E., S. K. Antiochos, and C. R. DeVore, A model for solar polar jets, Ap.J., 691, 61, 2009.

${ }^{41}$ Pariat, E., S. K. Antiochos, and C. R. DeVore, Three-dimensional modeling of quasi-homologous solar jets, Ap.J., 714, 1762, 2010.

${ }^{42}$ Edmondson, J. K., B. J. Lynch, S. K. Antiochos, C. R. DeVore, and T. H. Zurbuchen, Reconnection-driven dynamics of coronal-hole boundaries, Ap.J., 707, 1427, 2009.

${ }^{43}$ Edmondson, J. K., S. K. Antiochos, C. R. DeVore, B. J. Lynch, and T. H. Zurbuchen, Interchange reconnection and coronalhole dynamics, Ap.J., 714, 517, 2010a.

${ }^{44}$ Edmondson, J. K., S. K. Antiochos, C. R. DeVore, and T. H. Zurbuchen, Formation and dynamics of three-dimensional current sheets in the solar corona, Ap.J., 718, 72, $2010 \mathrm{~b}$.

${ }^{45}$ Karpen, J. T., C. R. DeVore, and S. K. Antiochos, Mechanisms for the onset and explosive eruption of coronal mass ejections and eruptive flares, Ap.J., 760, 81, 2012.

${ }^{46}$ Masson, S., S. K. Antiochos, and C. R. DeVore, A model for the escape of solar flare-accelerated particles, Ap.J., 771, 82, 2013.

${ }^{47}$ Chew, G. F., M. L. Goldberger, and F. E. Low, The Boltzmann equation and the one-fluid hydromagnetic equations in the absence of particle collisions, Proc. Roy. Soc. A, 236, 112, 1956.

${ }^{48}$ Hellinger, P., P. Trávniček, J. C. Kasper, A. J. Lazarus, Solar wind proton temperature anisotropy: Linear theory and WIND/SWE observations, Geophys. Res. Lett., 33, L09101, 2006.

${ }^{49}$ Krall, N. A. and A. W. Trivelpiece, Principles of Plasma Physics (New York: McGraw-Hill), 1973.

${ }^{50}$ Ilin, A. V., F. R. Chang Díaz, J. P. Squire, A. G. Tarditi, and M. D. Carter, Improved simulation of the ICRF waves in the VASIMR plasma, Computer Phys. Communications, 164, 251-257, 2004.

${ }^{51}$ Loureiro, N. F., A. A. Schekochihin, and S. C. Cowley, Instability of current sheets and formation of plasmoid chains, Phys. Plasmas, 14, 100703, 2007. 
${ }^{52}$ Bhattacharjee, A., Y.-M. Huang, H. Yang, and B. Rogers, Fast reconnection in high-Lundquist-number plasmas due to the plasmoid instability, Phys. Plasmas, 16, 112102, doi:10.1063/1.3264103, 2009.

${ }^{53}$ Daughton, W., Roytershteyn, V., Albright, B. J., Karimabadi, H., Yin, L., \& Bowers, K. J., Transition from collisional to kinetic regimes in large-scale reconnection layers, Phys. Rev. Lett., 103, 065004, 2009.

${ }^{54}$ Sheeley, N. R., Jr., et al., Measurements of flow speeds in the corona between 2 and $30 R_{\mathrm{s}}$, Ap.J., 484, $472,1997$.

${ }^{55}$ Wang, Y.-M., N. R. Sheeley Jr., D. G. Socker, R. A. Howard, and N. B. Rich, The dynamical nature of coronal streamers, J. Geophys. Res., 105, 25133, 2000.

${ }^{56}$ Sheeley, N. R., Jr. and Y.-M. Wang, Characteristics of coronal inflows, Ap.J., 579, 874, 2002.

${ }^{57}$ Sheeley, N. R., Jr. and Y.-M. Wang, In/out pairs and the detachment of coronal streamers, Ap.J., 655, 1142, 2007.

${ }^{58}$ Sheeley, N. R., Jr., D. D.-H. Lee, K. P. Casto, Y.-M. Wang, and N. B. Rich, The structure of streamer blobs, Ap.J., 694, 1471, 2009.

${ }^{59}$ Sheeley, N. R., Jr. and A. P. Rouillard, Tracking streamer blobs into the Heliosphere, Ap.J., 715, 300, 2010.

${ }^{60}$ Rouillard, A. P., et al., Intermittent release of transients in the slow solar wind: I. Remote sensing observations, J. Geophys. Res., 115, A04103, 2010a.

${ }^{61}$ Kilpua, E. K. J., et al., Small solar wind transients and their connection to the large-scale coronal structure, Sol. Phys., 256, $327,2009$.

${ }^{62}$ Rouillard, A. P., et al., Intermittent release of transients in the slow solar wind: II. In situ evidence, J. Geophys. Res., 115, A04104, 2010b. 